\title{
A Generalized Golden Rule Representative Value for Multiple-Criteria Decision Analysis
}

\author{
Zeyi Liu $^{(}$, Fuyuan Xiao ${ }^{(}$, Chin-Teng Lin $^{\circledR}$, Fellow, IEEE, Byeong Ho Kang ${ }^{\circledR}$, Senior Member, IEEE, \\ and Zehong $\mathrm{Cao}^{\circledR}$, Member, IEEE
}

\begin{abstract}
Multicriteria decision analysis evaluates multiple conflicting criteria in decision making, but conflicting criteria are typical in evaluating options. As the existing ordering operations involved in multicriteria decision making cannot easily be implemented with intervals, we assume that scalar representative values with intervals can effectively avoid this issue. To deal with interval-valued criteria, we propose a generalized golden rule representative value approach, which involves the sigmoid function of backpropagation neural networks to tune parameters. Our approach considers the uncertainties and side effects of the interval variables to improve individual scalar representative values. Based on numerical examples, we address the effectiveness of the proposed approach, and we provide a specific application concerning multicriteria decision making with interval criteria satisfaction.
\end{abstract}

Index Terms-Comparison system, golden rule representative value, interval valued, multicriteria decision function, sigmoid function, uncertainty.

\section{INTRODUCTION}

$\mathbf{M}$ CDM has been paid close attention in information systems in recent years [1]-[3]. The applications of MCDM have been widely discussed in many fields, such as supplier selection [4], [5], fault diagnosis [6], [7], and heartcare management [8], [9]. To make the decision outcome reliable, some techniques are applied to its optimization processes, including analytical hierarchy process (AHP) [10]-[12], decision making trial and evaluation laboratory (DEMATEL) method [13], [14], and technique for order preference by similarity to an ideal solution (TOPSIS) method [15]-[17]. The estimation outcome is assessed by combining the experts' synthesized opinions, it is vital to consider the uncertain

Manuscript received April 2, 2019; accepted May 10, 2019. This work was supported by the Chongqing Overseas Scholars Innovation Program under Grant cx2018077. This paper was recommended by Associate Editor Y.-P. Huang. (Corresponding author: Fuyuan Xiao.)

Z. Liu and F. Xiao are with the School of Computer and Information Science, Southwest University, Chongqing 400715, China (e-mail: doctorxiaofy@hotmail.com).

C.-T. Lin is with the Center for Artificial Intelligence, Faculty of Engineering and IT, University of Technology Sydney, Sydney, NSW 2007, Australia (e-mail: chin-teng.lin@uts.edu.au).

B. H. Kang and Z. Cao are with the Discipline of ICT, School of Technology, Environments and Design, University of Tasmania, Hobart, TAS 7001, Australia (e-mail: byeong.kang@utas.edu.au; zehong.cao@utas.edu.au).

Color versions of one or more of the figures in this paper are available online at http://ieeexplore.ieee.org.

Digital Object Identifier 10.1109/TSMC.2019.2919243 information in the learning process. In this case, existing studies addressed effective techniques to handle the uncertainty better, such as Dempster-Shafer evidence theory (DSET) [18]-[20], information entropy theory [21], [22], intuitionistic fuzzy set (IFS) theory [23]-[25], and hesitant fuzzy set (HFS) theory [26]. A multicriteria decision function can be used to aggregate the satisfaction of each alternative with respect to the individual criteria to obtain the alternative overall satisfaction to a decision problem [27], [28]. In addition, the interval value allows to represent uncertainty information, which attaches great importance to decision marking in a dynamic environment [29], [30]. For instance, the intervalvalued Pythagorean fuzzy set (IVPFS) [31] or interval-valued HFS (IFHFS) [17] can be considered as an effective extended model. Thus, if the individual criteria satisfaction by all alternatives is provided in terms of an interval value, reasonably obtaining the ordering is regarded as a very essential issue.

Traditionally, the method to measure the size of the interval simply uses the midpoint $m$. However, in some special situations, this method will lead to some misunderstandings. Although the interval range is very large, as long as the interval median points are the same, the traditional midpoint method results indicate that their scalar is the same. Therefore, the midpoint method is not considered appropriately for the interval ranges. Many scholars have discussed this problem [32]-[35]. Compared with other methods, the intervals are generally converted into representative values and then ordered by comparing their scalar values.

The interval midpoint and its range are the two most important eigenvalues for any interval; thus, the representative value of an interval mainly focuses on these two factors. Yager [36], [37] proposed a rule-based golden rule approach to obtain the representative value of the interval that can implement the required ordering and comparing operations when interval-valued information is given. Moreover, it can be seen that the result of the Sugeno integral [38], [39] is a special case of this model. However, while the representative values obtained according to this method can reflect some important features of the interval, they ignore the relative influence between the intervals in the comparison system and the uncertainty caused by the interval representation.

Considering the above problems, we introduce the sigmoid function [40] and the Takagi, Sugeno, and Kang (TSK) fuzzy model [41], which is commonly applied in biology and information science, to build an improved model for dealing with such situations. Due to its special characters, the sigmoid 
function has been focused on evolving fields, such as machine learning and neural networks. The TSK fuzzy model is one of the most famous fuzzy systems with a good nonlinear approximation ability and is widely used in many applications, such as system identification, pattern recognition, and image processing. In this paper, a new defined formula based on the sigmoid function is proposed to better express the correction relationship of the impact on the range. By introducing some new parameters to represent the inherent uncertain information brought by the range of the interval, the proposed method can be used to dynamically compare different intervals with more reasonable effects.

The rest of this paper is organized as follows. In Section II, some basic concepts about the TSK fuzzy model, the sigmoid function, and the golden rule representative value are introduced. In Section III, a new method for ordering the intervals based on the rule-based approach is proposed. In Section IV, some numerical examples are shown to discuss several different cases and to show the effectiveness of the approach. In Section V, some applications with interval-valued criteria satisfaction for the alternatives are shown to illustrate the practicality in multicriteria decision-making problems. In Section VI, the comparisons and discussion are given. And in Section VII, the sensitivity analysis is utilized to illustrate the robustness and sensitiveness. Finally, in Section VIII, some conclusions of the proposed method are drawn.

\section{PRELIMINARIES}

In this section, some basic concepts which include the golden rule, the TSK fuzzy model, and the sigmoid function are introduced.

\section{A. TSK Fuzzy Model}

The TSK fuzzy system, first proposed by Takagi and Sugeno [41], has a good nonlinear approximation ability and is widely used in many fields, such as system identification, classification [42], [43], and image processing [44]-[46]. In addition, some bionic algorithms are also widely used in fuzzy system modeling [47]. However, the TSK fuzzy model has always been regarded as one of the most famous fuzzy systems defined by the $I F$-Then rule. Some of the basic concepts are as follows.

Definition 1: The basic form of the TSK fuzzy model is shown as follows [41]:

$$
\begin{aligned}
& R_{i} \text { : If } x_{1} \text { is } A_{i 1}, x_{2} \text { is } A_{21} \text { and } \cdots x_{j} \text { is } A_{i j} \text { and } \cdots x_{n} \text { is } A_{i n} \\
& \text { Then, } y_{i}=f_{i}(X)
\end{aligned}
$$

where $X=x_{1}, x_{2}, \ldots, x_{n}$ are the predecessor variables and $A_{i j}$ is the fuzzy set of $x_{j}$ in Rule $i . f_{i}(X)$ represents the postcomponent variables. The specific expression is shown as follows:

$$
f_{i}(x)=a_{0}^{i}+a_{1}^{i} x_{1}+\cdots+a_{n}^{i} x_{n}
$$

where $R$ numerically expresses the total number of rules.

Definition 2: When the input of $X$ satisfies the condition that $X=\left[x_{1}, x_{2}, \ldots, x_{n}\right]$, the product method is introduced for $T$ norms to the predecessor variables. In addition, we use the center-of-gravity method to defuzzify. According to the above, the output of the TSK fuzzy model is expressed as [41]

$$
y=\frac{\sum_{i=1}^{R} \prod_{j=1}^{n} A_{i j}\left(x_{j}\right) y_{i}}{\sum_{i=1}^{R} \prod_{j=1}^{n} A_{i j}\left(x_{j}\right)} .
$$

Since the TSK fuzzy model was proposed, it has been shown to have broad application prospects in the field of state estimation due to its characteristics.

The conventional forecasting method has difficulty in meeting the actual requirements of the site for the following reasons.

1) The changes caused by the situation in the future cannot be completely grasped in advance.

2) Even though we know that certain complex factors have an impact on the system, it is still difficult to obtain quantitatively and to accurately determine its impact.

3) Many practical problems depend on the judgment ability and experience of the forecasters. The traditional methods without artificial intelligence technology require manual intervention to meet the actual forecasting requirements.

Compared with the traditional method, the TSK fuzzy model is based on rules. We can use the data information such as error and expert knowledge as the basic information, which provides flexibility for designing appropriate correction subsystems in prediction systems. Thus, the TSK fuzzy model is widely used in many applications.

\section{B. Golden Rule Representative Value}

To model complexity in the real world, many methods, such as complex networks [48], [49], nature-inspired methods [50]-[53], and neural networks [54], [55] have been presented. Additionally, using some existing rules, such as the golden rule is an efficient method for modeling complexity in the real world. In this section, the golden rule model presented by Yager is briefly introduced. The intervals often play an important role in many situations, especially in MCDM. In anticipation of solving the MCDM problems in situations in which the satisfaction of individual criteria by an alternative $X$ is provided in terms of an interval value from the unit interval rather than a precise value, the problem of ordering them must be considered [39].

Thus, to obtain the required ordering in the case of interval-valued information, the golden rule was introduced by Yager [37] to provide a scalar representative value for these intervals.

Definition 3: The golden rule representative value is based on some different rules; this is also called a rule-based description. Basically, all the representative values should be in the unit interval, namely, $\operatorname{Rep}(X) \in[0,1]$. To better explain its meaning, Yager subsequently implemented the golden rule using the Takagi-Sugeno approach to fuzzy system modeling [41], [56]. To obtain the desired representative value, the following four rules are proposed with the mean and range, which are the core variables of normal intervals [37].

1) If the mean of $X$ is large and the range of $X$ is large, then $\operatorname{Rep}(X)=1$. 
2) If the mean of $X$ is large and the range of $X$ is small, then $\operatorname{Rep}(X)=0.5$.

3) If the mean of $X$ is small and the range of $X$ is large, then $\operatorname{Rep}(X)=0.5$.

4) If the mean of $X$ is small and the range of $X$ is small, then $\operatorname{Rep}(X)=0$.

The large and small variables used in the rules above are represented as the linear fuzzy sets $L$ and $S$ defined on the unit interval. Thus, $L$ and $S$ are expressed as

$$
L(y)=y, S(y)=1-y .
$$

Definition 4: Based on the linear fuzzy sets, the TSK approach is used to obtain the representative value associated with an interval value $V$ and its mean $m$ and range $r$; namely, for a unique interval $V=[a, b]$, the specific function is shown as [39]

$$
\begin{aligned}
m & =\frac{a+b}{2} \\
r & =b-a \\
\operatorname{Rep}(V) & =\frac{1 m(1-r)+(0.5) m r+(0.5)(1-m) r+0(1-m)(1-r)}{m(1-r)+m r+(1-m) r+(1-m)(1-r)} .
\end{aligned}
$$

The result with the simplification operation is

$$
\operatorname{Rep}(V)=m+(0.5-m) r
$$

Then, we can discuss the formula. If $m<0.5$, the range tends to increase the representative value. In addition, if $m>$ 0.5 , the range tends to decrease the representative value. While if $m=0.5$, the representative value is equal to 0.5 .

However, $([\partial \operatorname{Rep}(V)] / \partial m)=1-r \geq 0$. Hence, the representative value always increases as the mean increases; it is monotonic with respect to the mean.

For an interval $V=[a, b]$, the representative value formula can be expressed in terms of $a$ and $b$ as

$$
\operatorname{Rep}(V)=\frac{1}{2}\left(a^{2}+2 b-b^{2}\right) .
$$

Specifically, when $m=0$, the golden rule will be degenerate into the traditional median method.

\section{Sigmoid Function}

The sigmoid function is a well-known function commonly found in biology and is also called the S-type growth curve. In information science, the sigmoid function is often used as a threshold function of a neural network due to its monotonically increasing character and its inverse function. Moreover, the variable is mapped between 0 and 1 [40].

To fully use this kind of function, some researchers have divided the sigmoid function into two parts. The first part is frequently called the log-sigmoid function, and the second part is usually expressed as the tan-sigmoid function. Additionally, both functions are continuous, monotonically increasing, and numerical functions. Here, we simply introduce some of their basic concepts.

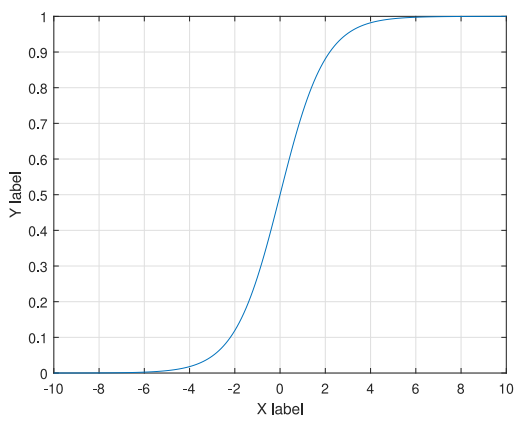

Fig. 1. Schematic of the log-sigmoid function.

1) Log-Sigmoid Function: According to its excellent and special characters, the log-sigmoid function has been applied in many fields, such as machine learning and neural networks [57], [58].

Definition 5: The basic equation of the log-sigmoid function is shown as follows [59]:

$$
f(x)=\frac{1}{1+e^{-x}} .
$$

This kind of function has very excellent characteristics in that the domain is the range of real numbers, but the value field is always in the unit interval $(0,1)$. Additionally, the derivative can be calculated by its own value, which is expressed as

$$
f^{\prime}(x)=f(x)(1-f(x))=\frac{e^{-x}}{\left(1+e^{-x}\right)^{2}} .
$$

The image corresponding to the function expression is shown in Fig. 1.

The log-sigmoid function can be seen everywhere in the field of machine learning. For example, we commonly use it as the response activation function of the output unit in the logistic regression and softmax regression classifiers [60]. The neuron activation function in the neural network is also widely used.

2) Tan-Sigmoid Function: Accordingly, there is also a tansigmoid function, which is widely used in backpropagation (BP) neural networks [61], [62].

Definition 6: In a BP neural network, the tan-sigmoid function is often used as a transfer function for the hidden layer and the output layer. The basic expression is shown as follows [63]:

$$
\theta(x)=\frac{e^{s}-e^{-s}}{e^{s}+e^{-s}}=\frac{2}{1+e^{-2 x}}-1 .
$$

Similarly, the derivative of the tan-sigmoid function also has a certain correlation with its own value, which is simply expressed as

$$
\theta^{\prime}(x)=1-\theta^{2}(x)
$$

The image corresponding to the function expression is shown in Fig. 2.

When constructing the correlation model of the neural network, the above two types of sigmoid functions are often expressed as the conversion functions of the output layer and the hidden layer. They can map the input of the domain in the real set to the interval $(0,1)$ and $(-1,1)$. The function 


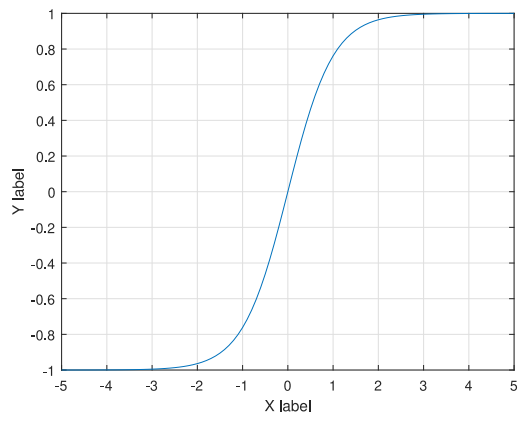

Fig. 2. Schematic of the tan-sigmoid function.

used is usually determined by the relationship between input and output. When the output cannot have a negative value, the log-sigmoid function is used. When the output contains a negative value, it is more appropriate to use the tan-sigmoid function.

\section{Generalized Golden RULE}

In this section, a generalized golden rule method is proposed to obtain the ordering of different intervals. Based on the basic idea of the golden rule representative value proposed by Yager [37], the sigmoid function is introduced to the process of dynamic comparison to obtain the ordering of different intervals.

Assume that there are several intervals $V_{i}=[a, b]$ in the comparison system in which their scalar values need to be compared to identify a reasonable order. In addition, all of the scalar values satisfy the condition that $a, b \in[0,1]$ and $a \leq b$. To explain the problem more intuitively, the notation $V_{1} \leq V_{2}$ is used to express the relationship that $V_{1}$ is larger than $V_{2}$.

For an interval, the mean value is widely considered to be the most fundamental variable. Therefore, the core idea is to consider the influence of the range factor on the basis of the mean to more reasonably measure the scalar value of the intervals.

Moreover, when the interval is applied to MCDM that represents the satisfaction of an alternative, the interval often comes with some uncertain information that is incidentally introduced by the decision maker. For example, assuming that alternative $x$ under a condition $A$ is considered to be $C_{A}(x)=[0.2,0.4]$, and another alternative $y$ is considered to be $C_{A}(y)=[0.2,0.8]$, then it is clear that the uncertainty of the evaluation for alternative $x$ is lower than alternative $y$; namely, when evaluating the above two alternatives, the evaluation of alternative $x$ is more convincing, while the result of alternative $y$ is relatively less certain.

Considering the factors mentioned above, the measurement of uncertainty absolutely needs to be introduced for the representative value of an interval. Therefore, under the premise of fully considering the uncertainty of each interval, it is more objective and more reasonable to obtain the representative values of multiple participating objects and to make relevant comparisons, which can also be seen as a kind of dimensionality reduction process.

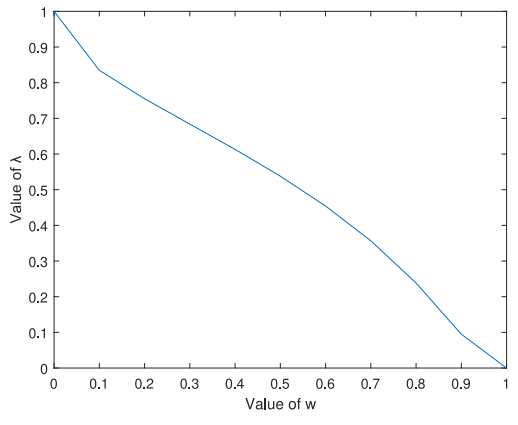

Fig. 3. Schematic of the relationship between $\lambda$ and $w$.

In the following, a rule-based description of a representative value for the comparison of intervals is introduced and is subsequently implemented with the TSK fuzzy system model and the sigmoid function.

Assume that $n$ intervals exist that need to be ordered using $V_{1}$ to $V_{n}$ to express. By performing correlation operations on each interval, their respective mean and range can be easily obtained. For instance, assume that we have $V_{i}=\left[a_{i}, b_{i}\right]$, then $m_{i}=\left[\left(a_{i}+b_{i}\right) / 2\right]$ and $r_{i}=b_{i}-a_{i}$; namely, there are $n$ intervals in the comparison system.

To consider the uncertain factor of range, we should first focus on the range of all comparison intervals. Thus, a weight determination is proposed to measure the size relationship of all the range values. For interval $V_{i}$, the weight is determined as follows:

$$
w_{i}=\frac{r_{i}}{\sum_{j=1}^{n} r_{j}}
$$

which satisfies the basic conditions that $w_{i} \in[0,1]$ and $\sum_{i=1}^{n} w_{i}=1$.

Since the comparison values in the comparison system are different when comparing, the weights assigned are not the same. Moreover, even if the same interval is compared with other different intervals, the weighted results are not always the same. For example, compare an interval $A$ with the remaining intervals $B, C$, and $D$ and derive a weighted result. Then, compare the interval $A$ with the intervals $B$ and $C$ to obtain a weight value with respect to the interval $A$. In this situation, unless the interval $D$ is an exact value, that is, its range is 0 , the weighting result of the interval $A$ obtained by the two comparison processes is different.

Then, after the weight determination process is completed, a new variable to express the range information is proposed as follows:

$$
r_{i}^{\prime}=\frac{2}{1+e^{\sqrt{\frac{w_{i}}{1-w_{i}}}}} r_{i} .
$$

The given determination is introduced based on the sigmoid function, which is widely used in BP neural networks. According to the above conditions, the size of $w_{i}$ is clearly within the range $[0,1]$. To better show the relationship between the range $r_{i}$ and the new variable $r_{i}^{\prime}$, the coefficient of $r_{i}$ can be seen as a variable $\lambda_{i}$ with respect to the size of the range weight for the interval $V_{i}$. Moreover, to represent the effect of 
this parameter on the range value better, the law of change for $\lambda_{i}$ is shown in Fig. 3.

As shown in the above figure, the following conclusions can be clearly drawn. Taking the interval $V_{i}$ as an example, the size of the new variable $r_{i}^{\prime}$ satisfies the following laws.

1) The greater the $r_{i}$, the more comparatively uncertain it is. When $r_{i}$ is extremely large, it is extremely uncertain. When $r_{i}$ is equal to 1 , it means that it is completely uncertain.

2) The smaller the $r_{i}$, the more comparatively certain it is. When $r_{i}$ is extremely small, it is extremely certain. When $r_{i}$ is equal to 0 , it means that it is completely certain.

When the number of members in the comparison system is fixed, if the range of one of the intervals is extended, the corresponding weight $w_{i}$ also increases, and the value of the variable $\lambda_{i}$ decreases, which can be interpreted as the range of the interval is increased to make it more uncertain, and the reduction in the variable $\lambda_{i}$ can suppress the effect of the range increase to some extent.

Conversely, when the interval of the interval decreases, the weight occupied in the comparison system decreases, then the variable $\lambda_{i}$ increases. It can also be interpreted as the interval of the interval decreases, making it more deterministic, and the increase in the variable $\lambda_{i}$ can be expressed to some extent by the effect of increasing the variation in the range.

In the following, the concepts of mean $m_{i}$ and the new range $r_{i}^{\prime}$ associated with an interval value are used to obtain the representation value. In addition, the following four rules are introduced for measuring our desired results.

Rule $_{1}$ : If the mean $m_{i}$ is large, and the new range $r_{i}^{\prime}$ is small, then $\operatorname{Rep}(V)=1$.

Rule $e_{2}$ If the mean $m_{i}$ is large, and the new range $r_{i}^{\prime}$ is large, then $\operatorname{Rep}(V)=0.5$.

Rule $_{3}$ : If the mean $m_{i}$ is small, and the new range $r_{i}^{\prime}$ is large, then $\operatorname{Rep}(V)=0.5$.

Rule 4: If the mean $m_{i}$ is small, and the new range $r_{i}^{\prime}$ is small, then $\operatorname{Rep}(V)=0$.

Here, large is represented by linear fuzzy sets $L$, and small is represented by $S, L(x)=x, S(x)=1-x$, which are all defined on the unit interval. Using the TSK approach to model the rule-based description of the generalized golden rule representative value (GGRV) associated with the interval value $V_{i}$, the following equation can be obtained:

$$
\begin{aligned}
& \operatorname{Rep}\left(V_{i}\right) \\
& =\frac{1 m_{i}\left(1-r_{i}^{\prime}\right)+(0.5) m_{i} r_{i}^{\prime}+(0.5)\left(1-m_{i}\right) r_{i}^{\prime}+0\left(1-m_{i}\right)\left(1-r_{i}^{\prime}\right)}{m_{i}\left(1-r_{i}^{\prime}\right)+m_{i} r_{i}^{\prime}+\left(1-m_{i}\right) r_{i}^{\prime}+\left(1-m_{i}\right)\left(1-r_{i}^{\prime}\right)} .
\end{aligned}
$$

Performing the proper algebra, the final equation is expressed as follows:

$$
\operatorname{Rep}\left(V_{i}\right)=m_{i}+(0.5-m) r_{i}^{\prime} .
$$

According to the above equation, if $m_{i}<0.5$, the influence of $r_{i}^{\prime}$ is positive and tends to increase the representative value. In addition, if $m>0.5$, the influence of $r_{i}^{\prime}$ is negative and tends to decrease the representative value. Specifically, if $m=0.5$, the representative value $\operatorname{Rep}\left(V_{i}\right)=0.5$, which is independent of the uncertainty and the influence of $r_{i}{ }^{\prime}$.
TABLE I

Calculation Results in Case 1

\begin{tabular}{cccccccc}
\hline Interval & $\mathrm{m}$ & $\mathrm{r}$ & $\mathrm{w}$ & $\lambda$ & $\mathrm{r}$ & Golden rule & Proposed method \\
\hline$[0.1,0.4]$ & 0.2500 & 0.3000 & 0.3333 & 0.6605 & 0.1981 & 0.3250 & 0.2995 \\
{$[0.2,0.5]$} & 0.3500 & 0.3000 & 0.3333 & 0.6605 & 0.1981 & 0.3950 & 0.3797 \\
{$[0.3,0.6]$} & 0.4500 & 0.3000 & 0.3333 & 0.6605 & 0.1981 & 0.4650 & 0.4599 \\
\hline
\end{tabular}

TABLE II

Calculation Results in Case 2

\begin{tabular}{cccccccc}
\hline Interval & $\mathrm{m}$ & $\mathrm{r}$ & $\mathrm{w}$ & $\lambda$ & $\mathrm{r}$ & Golden rule & Proposed method \\
\hline$[0.4,0.7]$ & 0.5500 & 0.3000 & 0.2500 & 0.7191 & 0.2157 & 0.5350 & 0.5392 \\
{$[0.5,0.8]$} & 0.6500 & 0.3000 & 0.2500 & 0.7191 & 0.2157 & 0.6050 & 0.6176 \\
{$[0.6,0.9]$} & 0.7500 & 0.3000 & 0.2500 & 0.7191 & 0.2157 & 0.6750 & 0.6961 \\
{$[0.7,1.0]$} & 0.8500 & 0.3000 & 0.2500 & 0.7191 & 0.2157 & 0.7450 & 0.7745 \\
\hline
\end{tabular}

Moreover, the representative value can be proven monotonic with respect to the mean $m_{i}$

$$
V_{i}^{\prime}=\frac{\partial \operatorname{Rep}\left(V_{i}\right)}{\partial m}=1-r_{i}^{\prime} \geq 0 .
$$

Thus, the representative value always increases as the mean $m_{i}$ increases. Based on the representative value equation, to find the boundary condition and some properties.

1) When $r_{i}=0$, regardless of what other intervals in the system are compared, $r_{i}^{\prime}$ is equal to 0 . In addition, the variable $\lambda_{i}$ is the maximum value 1 , and the generalized golden rule degenerates to the midpoint method.

2) When an interval in which the range is nonzero is compared with another interval in which the range is 0 , the representative value degenerates to the midpoint method.

The flowchart of the calculation process is shown in Fig. 4.

\section{NUMERICAL EXAMPLES}

In this section, some specific numerical examples of the calculation with some explanations are illustrated to show the whole process of our proposed method.

Example 1:

Case 1: Assume that there are three intervals, $V_{1}=\left[a_{1}, b_{1}\right]$, $V_{2}=\left[a_{2}, b_{2}\right]$, and $V_{3}=\left[a_{3}, b_{3}\right]$, where $V_{1}=[0.1,0.4]$, $V_{2}=[0.2,0.5]$, and $V_{3}=[0.3,0.6]$. After calculation, the following results can be obtained in Table I.

Case 2: For $V_{4}=[0.4,0.7], V_{5}=[0.5,0.8], V_{6}=$ $[0.6,0.9]$, and $V_{7}=[0.7,1.0]$, the following results can be obtained in Table II.

Obviously, when the original range between the comparison regions is equal but the respective mean is different, the obtained GGRV is not much different from the golden rule method, and the size order relationship can be correctly reflected.

Moreover, by comparing the results in cases 1 and 2, it is shown that when the interval ranges are the same, the larger mean indicates that the greater the overall support, the smaller the difference between the results of the proposed method and the results of the golden rule method. The results also showed that the difference is greater than the results of the golden rule method.

Example 2: Assume that there are five intervals, $V_{1}=$ $\left[a_{1}, b_{1}\right], V_{2}=\left[a_{2}, b_{2}\right], V_{3}=\left[a_{3}, b_{3}\right], V_{4}=\left[a_{4}, b_{4}\right]$, and $V_{5}=\left[a_{5}, b_{5}\right]$, where $V_{1}=[0.1,0.1], V_{2}=[0.1,0.3]$, $V_{3}=[0.1,0.5], V_{4}=[0.1,0.7]$, and $V_{5}=[0.1,0.9]$. After the 


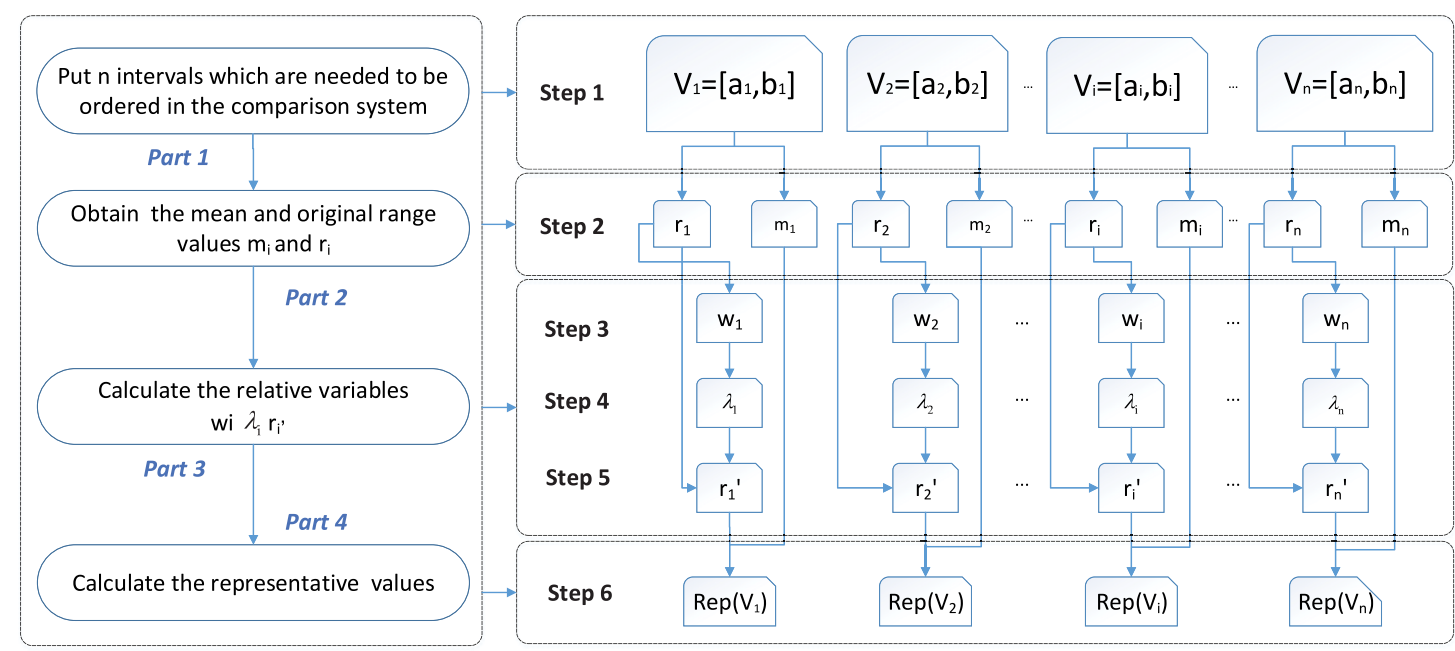

Fig. 4. Flowchart of the calculation process.

TABLE III

Calculation Results in Example 2

\begin{tabular}{cccccccc}
\hline Interval & $\mathrm{m}$ & $\mathrm{r}$ & $\mathrm{w}$ & $\lambda$ & r' & Golden rule & Proposed method \\
\hline$[0.1,0.1]$ & 0.1000 & 0.0000 & 0.0000 & 1.0000 & 0.0000 & 0.1000 & 0.1000 \\
{$[0.1,0.3]$} & 0.2000 & 0.2000 & 0.1000 & 0.8349 & 0.1670 & 0.2600 & 0.2501 \\
{$[0.1,0.5]$} & 0.3000 & 0.4000 & 0.2000 & 0.7551 & 0.3020 & 0.3800 & 0.3604 \\
{$[0.1,0.7]$} & 0.4000 & 0.6000 & 0.3000 & 0.6839 & 0.4103 & 0.4600 & 0.4410 \\
{$[0.1,0.9]$} & 0.5000 & 0.8000 & 0.4000 & 0.6130 & 0.4904 & 0.5000 & 0.5000
\end{tabular}

TABLE IV

CALCULATION Results in EXAMPLE 3

\begin{tabular}{cccccccc}
\hline Interval & $\mathrm{m}$ & $\mathrm{r}$ & $\mathrm{w}$ & $\lambda$ & r' & Golden rule & Proposed method \\
\hline$[0.5,0.5]$ & 0.5000 & 0.0000 & 0.0000 & 1.0000 & 0.0000 & 0.5000 & 0.5000 \\
{$[0.4,0.6]$} & 0.5000 & 0.2000 & 0.1000 & 0.8349 & 0.1670 & 0.5000 & 0.5000 \\
{$[0.3,0.7]$} & 0.5000 & 0.4000 & 0.2000 & 0.7551 & 0.3020 & 0.5000 & 0.5000 \\
{$[0.2,0.8]$} & 0.5000 & 0.6000 & 0.3000 & 0.6839 & 0.4103 & 0.5000 & 0.5000 \\
{$[0.1,0.9]$} & 0.5000 & 0.8000 & 0.4000 & 0.6130 & 0.4904 & 0.5000 & 0.5000 \\
\hline
\end{tabular}

process of calculation, the following results can be obtained in Table III.

Clearly, the values of the left endpoints of the five intervals in the comparison system are the same in Example 2, but the ranges are different. The calculated results are shown above, which shows that the representative value increases as the interval mean increases. Specifically, when the mean of the interval is 0.5 or the interval range is 0 , the proposed method degenerates to the midpoint method, which is the same as the golden rule method.

Example 3: Assume that there are five intervals, $V_{1}=$ $\left[a_{1}, b_{1}\right], V_{2}=\left[a_{2}, b_{2}\right], V_{3}=\left[a_{3}, b_{3}\right], V_{4}=\left[a_{4}, b_{4}\right]$, and $V_{5}=\left[a_{5}, b_{5}\right]$, where $V_{1}=[0.5,0.5], V_{2}=[0.4,0.6]$, $V_{3}=[0.3,0.7], V_{4}=[0.2,0.8]$, and $V_{5}=[0.1,0.9]$. After calculation, the following results can be obtained in Table IV.

In the five intervals shown in Example 3, the values of the mean are all 0.5 , but each of them has a different range.

When the range is 0.5 , both the golden rule method and the proposed method degenerate to the midpoint method, which shows the calculation result of the representative value and the independence of the uncertainty information given by the range when $m=0.5$.

Example 4: Assume that there are five intervals, $V_{1}=$ $\left[a_{1}, b_{1}\right], V_{2}=\left[a_{2}, b_{2}\right], V_{3}=\left[a_{3}, b_{3}\right], V_{4}=\left[a_{4}, b_{4}\right]$, and
TABLE V

Calculation Results in EXAMPLe 4

\begin{tabular}{cccccccc}
\hline Interval & $\mathrm{m}$ & $\mathrm{r}$ & $\mathrm{w}$ & $\lambda$ & r' & Golden Rule & Proposed method \\
\hline$[0.0,0.9]$ & 0.4500 & 0.9000 & 0.3600 & 0.6416 & 0.5775 & 0.4950 & 0.4789 \\
{$[0.1,0.8]$} & 0.4500 & 0.7000 & 0.2800 & 0.6979 & 0.4885 & 0.4850 & 0.4744 \\
{$[0.2,0.7]$} & 0.4500 & 0.5000 & 0.2000 & 0.7551 & 0.3775 & 0.4750 & 0.4689 \\
{$[0.3,0.6]$} & 0.4500 & 0.3000 & 0.1200 & 0.8174 & 0.2452 & 0.4650 & 0.4623 \\
{$[0.4,0.5]$} & 0.4500 & 0.1000 & 0.0400 & 0.8983 & 0.0898 & 0.4550 & 0.4545 \\
\hline
\end{tabular}

$V_{5}=\left[a_{5}, b_{5}\right]$, where $V_{1}=[0.0,0.9], V_{2}=[0.1,0.8]$, $V_{3}=[0.2,0.7], V_{4}=[0.3,0.6]$, and $V_{5}=[0.4,0.5]$. After calculation, the following results can be obtained in Table V.

As shown in Example 4, although the median points of the five intervals are the same, they are not 0.5. Evidently, when the range is larger, the proposed method shows little difference from the golden rule. In addition, as the range is larger, the difference is also larger, and the smaller the range, the smaller the difference.

\section{APPLICATION}

In this section, some applications are analyzed to illustrate the practicality of the proposed method.

\section{A. Background Concepts of Interval-Valued Criteria Satisfactions}

For the following discussion, there exists some uncertainty in the determination of the satisfaction for a criteria $C_{i}$ by an alternative $x$ [39], [64]. Generally, the assessment provided by experts often meets certain uncertainty information. From a realistic point of view, the evaluation results expressed as intervals are more objective than the traditional single-value evaluation results. In some practical applications, interval satisfaction can usually avoid information loss due to excessive accuracy. Hence, the usage of interval satisfaction is quite essential in the real world.

To illustrate the problem better, the framework of the MCDM problems with satisfaction intervals is shown in Fig. 5.

\section{B. Application 1}

Assume that each $C_{i}(x)$ is expressed as an interval value, which denotes that $C_{i}(x)=\left[a_{i}(x), b_{i}(x)\right]$. The core work 


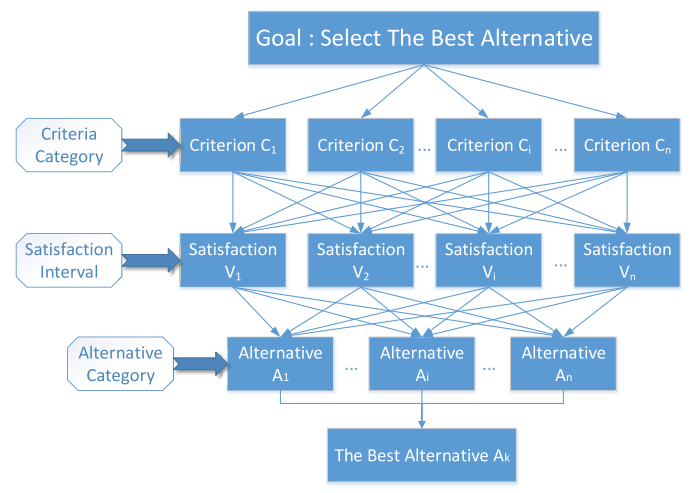

Fig. 5. Framework of the MCDM problems with satisfaction intervals.

TABLE VI

Calculation Results of the Relative Variables of $C_{i}(x)$

\begin{tabular}{cccccc}
\hline Interval & Index & $\mathrm{w}$ & $\lambda$ & r' & Proposed method \\
\hline$[0.2,0.7]$ & 1 & 0.5556 & 0.4928 & 0.2464 & 0.4623 \\
{$[0.4,0.8]$} & 2 & 0.4444 & 0.5804 & 0.2322 & 0.5768 \\
{$[0.5,0.5]$} & 3 & 0.0000 & 1.0000 & 0.0000 & 0.5000 \\
\hline
\end{tabular}

is to evaluate $D(x)=\operatorname{Max}\left[T\left(C_{\lambda(j)}(x), \mu\left(H_{j}\right)\right)\right]$, where $j=$ 1 to $q$. Moreover, if $C_{\lambda(j)}(x)=\left[a_{\lambda(j)}(x), b_{\lambda(j)}(x)\right]$, then $T\left(C_{\lambda(j)}(x), \mu\left(H_{j}\right)\right)$ can be calculated as follows [39]:

$T\left(C_{\lambda(j)}(x), \mu\left(H_{j}\right)\right)=\left[T\left(a_{\lambda(j)}(x), \mu\left(H_{j}\right)\right), T\left(b_{\lambda(j)}(x), \mu\left(H_{j}\right)\right)\right]$.

Suppose that we have three criteria, $C_{1}, C_{2}$, and $C_{3}$, and their respective satisfactions by an alternative $x$ are interval valued and are denoted by $C_{1}(x), C_{2}(x)$, and $C_{3}(x)$. Here, the specific values are given as follows:

$C_{1}(x)=[0.2,0.7], C_{2}(x)=[0.4,0.8], C_{3}(x)=[0.5,0.5]$.

Step 1: Obtain their mean values and respective original range values. The results are shown as follows:

$$
\begin{aligned}
& m_{1}(x)=\frac{0.2+0.7}{2}=0.45 \\
& m_{2}(x)=\frac{0.4+0.8}{2}=0.60 \\
& m_{3}(x)=\frac{0.5+0.5}{2}=0.50 \\
& r_{1}(x)=0.7-0.2=0.5 \\
& r_{2}(x)=0.8-0.4=0.4 \\
& r_{3}(x)=0.5-0.5=0.0 .
\end{aligned}
$$

Step 2: Calculate their representative values using the proposed method and obtain the order by comparing the calculated results for their representative values shown in Table VI.

Thus, we have $\lambda(1)=2, \lambda(2)=3$, and $\lambda(3)=1$, which means that $C_{2}(x)>C_{3}(x)>C_{1}(x)$.

Step 3: Obtain the result of $\mu\left(H_{j}\right)$. According to the above calculation results, $\left(H_{j}\right)$ can be expressed as

$$
\begin{aligned}
& H_{1}=\left\{C_{2}\right\} \\
& H_{2}=\left\{C_{2}, C_{3}\right\} \\
& H_{3}=\left\{C_{2}, C_{3}, C_{1}\right\} .
\end{aligned}
$$

TABLE VII

Calculation Results of THE Relative VARIABLES OF $T\left(C_{\lambda(j)}(x), \mu\left(H_{j}\right)\right)$

\begin{tabular}{cccccccc}
\hline Interval & Index & $\mathrm{m}$ & $\mathrm{r}$ & $\mathrm{w}$ & $\lambda_{i}$ & $\mathrm{r}$ & Representative value \\
\hline$[0.4,0.4]$ & 1 & 0.4000 & 0.0000 & 0.0000 & 1.0000 & 0.0000 & 0.4000 \\
{$[0.5,0.5]$} & 2 & 0.5000 & 0.0000 & 0.0000 & 1.0000 & 0.0000 & 0.5000 \\
{$[0.2,0.7]$} & 3 & 0.4500 & 0.5000 & 1.0000 & 0.0000 & 0.0000 & 0.4500 \\
\hline
\end{tabular}

TABLE VIII

SIS OF TEN SUPPLIERS

\begin{tabular}{cc|cc}
\hline Supplier & SIS & Supplier & SIS \\
\hline$S_{1}$ & {$[0.2,0.7]$} & $S_{6}$ & {$[0.4,0.5]$} \\
$S_{2}$ & {$[0.5,0.6]$} & $S_{7}$ & {$[0.5,0.7]$} \\
$S_{3}$ & {$[0.8,0.9]$} & $S_{8}$ & {$[0.2,0.5]$} \\
$S_{4}$ & {$[0.0,1.0]$} & $S_{9}$ & {$[0.2,0.6]$} \\
$S_{5}$ & {$[0.3,0.3]$} & $S_{10}$ & {$[0.3,0.6]$} \\
\hline
\end{tabular}

TABLE IX

CALCUlation Results in EXAMPLE 5

\begin{tabular}{cccccccc}
\hline Interval & $\mathrm{m}$ & $\mathrm{r}$ & $\mathrm{w}$ & $\lambda$ & $\mathrm{r}$ & Proposed method & Rank \\
\hline$[0.2,0.7]$ & 0.4500 & 0.5000 & 0.1667 & 0.7800 & 0.3900 & 0.4695 & 5 \\
{$[0.5,0.6]$} & 0.5500 & 0.1000 & 0.0333 & 0.9074 & 0.0907 & 0.5455 & 3 \\
{$[0.8,0.9]$} & 0.8500 & 0.1000 & 0.0333 & 0.9074 & 0.0907 & 0.8182 & 1 \\
{$[0.0,1.0]$} & 0.5000 & 1.0000 & 0.3333 & 0.6605 & 0.6605 & 0.5000 & 4 \\
{$[0.3,0.3]$} & 0.3000 & 0.0000 & 0.0000 & 1.0000 & 0.0000 & 0.3000 & 10 \\
{$[0.4,0.5]$} & 0.4500 & 0.1000 & 0.0333 & 0.9074 & 0.0907 & 0.4545 & 7 \\
{$[0.5,0.7]$} & 0.6000 & 0.2000 & 0.0667 & 0.8672 & 0.1734 & 0.5827 & 2 \\
{$[0.2,0.5]$} & 0.3500 & 0.3000 & 0.1000 & 0.8349 & 0.2505 & 0.3876 & 9 \\
{$[0.2,0.6]$} & 0.4000 & 0.4000 & 0.1333 & 0.8064 & 0.3225 & 0.4323 & 8 \\
{$[0.3,0.6]$} & 0.4500 & 0.3000 & 0.1000 & 0.8349 & 0.2505 & 0.4625 & 6 \\
\hline
\end{tabular}

TABLE $X$

Normalized Weights For All the Criteria

\begin{tabular}{cccccc}
\hline Criteria & $C_{1}$ & $C_{2}$ & $C_{3}$ & $C_{4}$ & $C_{5}$ \\
\hline$\omega$ & 0.30298 & 0.25329 & 0.17542 & 0.16883 & 0.09948 \\
\hline
\end{tabular}

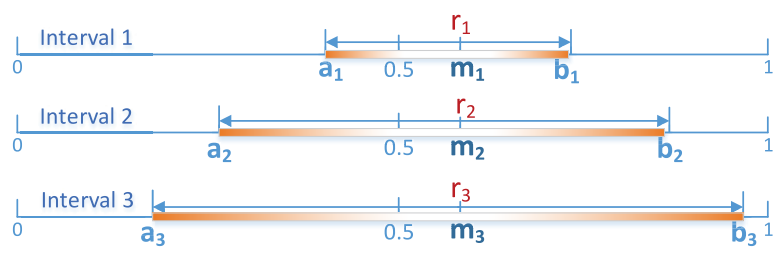

Fig. 6. Different intervals with the same mean value.

Additionally, we first assume that there exits the following fuzzy measure $\mu$ with respect to the given criteria [65], [66]:

$$
\begin{aligned}
\mu\left(\left\{C_{1}\right\}\right) & =0.3, \mu\left(\left\{C_{2}\right\}\right)=0.4, \mu\left(\left\{C_{3}\right\}\right)=0.3 \\
\mu\left(\left\{C_{1}, C_{2}\right\}\right) & =0.8, \mu\left(\left\{C_{1}, C_{3}\right\}\right)=0.5, \mu\left(\left\{C_{2}, C_{3}\right\}\right)=0.9 \\
\mu\left(\left\{C_{1}, C_{2}, C_{3}\right\}\right) & =1 .
\end{aligned}
$$

Thus, the following equation can be easily obtained with the given fuzzy measure:

$$
\begin{aligned}
& \mu\left(H_{1}\right)=\mu\left(\left\{C_{2}\right\}\right)=0.4 \\
& \mu\left(H_{2}\right)=\mu\left(\left\{C_{2}, C_{3}\right\}\right)=0.9 \\
& \mu\left(H_{3}\right)=\mu\left(\left\{C_{2}, C_{3}, C_{1}\right\}\right)=1 .
\end{aligned}
$$

Step 4: Calculate the value of $T\left(C_{\lambda(j)}(x), \mu\left(H_{j}\right)\right)$. In this situation, we simply assume that $T$ is the min norm. 
TABLE XI

Aggregated Normalized Interval-Valued Hesitant FuzZy Decision MatriX

\begin{tabular}{cccccc}
\hline$A_{i}$ & $C_{1}$ & $C_{2}$ & $C_{3}$ & $C_{4}$ & $C_{5}$ \\
\hline$A_{1}$ & {$[0.2348,0.3351]$} & {$[0.7379,0.8413]$} & {$[0.2000,0.3000]$} & {$[0.6366,0.7379]$} & {$[0.5358,0.6366]$} \\
$A_{2}$ & {$[0.2681,0.3684]$} & {$[0.6698,0.7711]$} & {$[0.2681,0.3684]$} & {$[0.7116,0.8183]$} & {$[0.6698,0.7711]$} \\
$A_{3}$ & {$[0.1680,0.2681]$} & {$[0.6698,0.7711]$} & {$[0.1347,0.2348]$} & {$[0.7379,0.8413]$} & {$[0.5783,0.6825]$} \\
\hline
\end{tabular}

To illustrate clearly, $B_{j}(x)$ is introduced, which satisfies the equation that $B_{j}(x)=T\left(C_{\lambda(j)}(x), \mu\left(H_{j}\right)\right)$. Thus, we can obtain that

$$
B_{j}(x)=\left[a_{\lambda(j)}(x) \wedge \mu\left(H_{j}\right), b_{\lambda(j)}(x) \wedge \mu\left(H_{j}\right)\right] .
$$

And

$$
\begin{aligned}
& B_{1}(x)=[0.4 \wedge 0.4,0.8 \wedge 0.4]=[0.4,0.4] \\
& B_{2}(x)=[0.5 \wedge 0.9,0.5 \wedge 0.9]=[0.5,0.5] \\
& B_{3}(x)=[0.2 \wedge 1.0,0.7 \wedge 1.0]=[0.2,0.7] .
\end{aligned}
$$

Step 5: Calculate the representative values using the proposed method to order the value of $T\left(C_{\lambda(j)}(x), \mu\left(H_{j}\right)\right)$ to obtain $D(x)$.

According to the proposed method, the calculation results can be shown in Table VII.

Thus, we can obtain the order that $B_{1}(x)<B_{3}(x)<B_{2}(x)$, and $D(x)=\operatorname{Max}\left[B_{1}(x), B_{2}(x), B_{3}(x)\right]=B_{2}(x)=[0.5,0.5]$ in this situation.

To illustrate the application process, the above process simply calculates an alternative $x$. However, in more complicated cases, there will be more possible alternatives $X=$ $\left[x_{1}, x_{2}, \ldots, x_{n}\right]$ for comparison. In that case, the maximum of $D(x)$ must be selected to obtain the best alternative.

\section{Application 2}

Manufacturer plans to purchase a batch of new equipment from the market. There are ten suppliers $\left\{S_{1}, S_{2}, \ldots, S_{10}\right\}$ are taken into consideration. Several experts consider them from four aspects, such as environment production, quality of equipment, production efficiency, and economy. Note that all the satisfaction provided by experts are considered as the form of interval. After the aggregation process, the synthesized interval satisfaction (SIS) are achieved, which are denoted as $V=\left\{V_{1}, V_{2}, \ldots, V_{10}\right\}$ and the details are shown in Table VIII.

After the calculation process, the following findings can be achieved, which are shown in Table IX. We can see that supplier $S_{3}$ could be preferentially considered as the best selection and supplier $S_{5}$ is possibly regarded as the worst one.

\section{COMPARISON AND DISCUSSION}

Traditionally, to measure the size of the interval, the midpoint method is used. However, using this method causes some misunderstandings in some special situations.

As shown in Fig. 6, the usage of the simple surrogate for the interval, in certain cases, may cause the situation that no fully feedbacks to the preferences for some decision makers. For example, we suppose the outcomes of an alternative $x_{i}$ with criteria $C_{i}$ are given by three experts as shown in Fig. 6. If decision makers use the traditional midpoint method to compare the results, they will be notified of the outcomes without
TABLE XII

Calculation Results for All the Green Suppliers

\begin{tabular}{cccc}
\hline Green supplier & Interval & GGRV & Rank \\
\hline$A_{1}$ & {$[0.4536,0.5548]$} & 0.5039 & 2 \\
$A_{2}$ & {$[0.4844,0.5861]$} & 0.5329 & 1 \\
$A_{3}$ & {$[0.4260,0.5274]$} & 0.4783 & 3 \\
\hline
\end{tabular}

completely consistent evaluation. Furthermore, it may cause serious information loss and lead to the unpredictable cost in some complicated application environments, if we only keep using the traditional midpoint method without the functions to measure uncertain information.

Therefore, Yager [37] proposed a rule-based approach to solving this problem named the golden rule representative value method. Although the golden rule method has many great advantages, the impact of the influence of other interval ranges is not considered in the comparison system with uncertain information. To address this issue, the proposed method collects the range information of all intervals in comparison system and then introduce the sigmoid function in BP neural networks to tune parameters. Compared with traditional approaches, the representative values of the individual interval are dynamically adjusted with the change of other intervals. In other words, for any intervals, the uncertain information is not only related to its range but also related to other intervals in comparison system, which can be seen as the relative uncertainty. Furthermore, the proposed method retains the basic idea of the golden rule with the usage of the TSK fuzzy model.

As shown in Section IV, the calculation results in many different cases are listed. Thus, it is simple to find the following.

1) The proposed method is completely data driven, and it extracts the implicit uncertain information based on the interval data. Thus, it considers the influence of the interval range on its representative value.

2) Taking into account the influence of the uncertainty of each interval in the comparison system, the proposed method embodies a process of dynamic comparison; that is, the representative value used to represent the interval will change when the interval for comparison is different.

3) Introducing the sigmoid function into the BP neural network can clearly indicate the uncertainty relationship reflected in the reasonable constraint effect of the parameter $\lambda$ on the range and well limit the range of the parameter.

4) Retaining the basic idea of the golden rule proposed by Yager and using the TSK fuzzy model to make the proposed method are consistent with the advantages. 
TABLE XIII

Sensitive Analysis Calculation Results in Section VII

\begin{tabular}{|c|c|c|c|c|c|c|c|c|c|c|c|}
\hline \multicolumn{4}{|c|}{ Case 1} & \multicolumn{4}{|c|}{ Case 2} & \multicolumn{4}{|c|}{ Case 3} \\
\hline$\epsilon$ values & Interval & GGRV & Rank & $\epsilon$ values & Interval & GGRV & Rank & $\epsilon$ values & Interval & GGRV & Rank \\
\hline \multirow{5}{*}{$\epsilon=0.00$} & {$[0.20,0.60]$} & 0.4245 & 1 & & {$[0.30,0.70]$} & 0.5000 & 1 & \multirow{5}{*}{$\epsilon=0.00$} & {$[0.40,0.80]$} & 0.5755 & 5 \\
\hline & {$[0.25,0.55]$} & 0.4205 & 2 & & {$[0.35,0.65]$} & 0.5000 & 1 & & {$[0.45,0.75]$} & 0.5795 & 4 \\
\hline & {$[0.30,0.50]$} & 0.4151 & 3 & $\epsilon=0.00$ & {$[0.40,0.60]$} & 0.5000 & 1 & & {$[0.50,0.70]$} & 0.5849 & 3 \\
\hline & {$[0.35,0.45]$} & 0.4083 & 4 & & {$[0.45,0.55]$} & 0.5000 & 1 & & {$[0.55,0.65]$} & 0.5917 & 2 \\
\hline & {$[0.40,0.40]$} & 0.4000 & 5 & & {$[0.50,0.50]$} & 0.5000 & 1 & & {$[0.60,0.60]$} & 0.6000 & 1 \\
\hline \multirow{5}{*}{$\epsilon=0.05 * r_{1}$} & {$[0.20-\epsilon, 0.60+\epsilon]$} & 0.4262 & 1 & & {$[0.30-\epsilon, 0.70+\epsilon]$} & 0.5000 & 1 & \multirow{5}{*}{$\epsilon=0.05 * r_{1}$} & {$[0.40-\epsilon, 0.80+\epsilon]$} & 0.5738 & 5 \\
\hline & {$[0.25,0.55]$} & 0.4207 & 2 & & {$[0.35,0.65]$} & 0.5000 & 1 & & {$[0.45,0.75]$} & 0.5792 & 4 \\
\hline & {$[0.30,0.50]$} & 0.4152 & 3 & $\epsilon=0.05 * r_{1}$ & {$[0.40,0.60]$} & 0.5000 & 1 & & {$[0.50,0.70]$} & 0.5848 & 3 \\
\hline & {$[0.35,0.45]$} & 0.4084 & 4 & & {$[0.45,0.55]$} & 0.5000 & 1 & & {$[0.55,0.65]$} & 0.5916 & 2 \\
\hline & {$[0.40,0.40]$} & 0.4000 & 5 & & {$[0.50,0.50]$} & 0.5000 & 1 & & {$[0.60,0.60]$} & 0.6000 & 1 \\
\hline \multirow{5}{*}{$\epsilon=0.05 * r_{2}$} & {$[0.20,0.60]$} & 0.4249 & 1 & & {$[0.30,0.70]$} & 0.5000 & 1 & \multirow{5}{*}{$\epsilon=0.05 * r_{2}$} & {$[0.40,0.80]$} & 0.5751 & 5 \\
\hline & {$[0.25-\epsilon, 0.55+\epsilon]$} & 0.4221 & 2 & & {$[0.35-\epsilon, 0.65+\epsilon]$} & 0.5000 & 1 & & {$[0.45-\epsilon, 0.75+\epsilon]$} & 0.5779 & 4 \\
\hline & {$[0.30,0.50]$} & 0.4152 & 3 & $\epsilon=0.05 * r_{2}$ & {$[0.40,0.60]$} & 0.5000 & 1 & & {$[0.50,0.70]$} & 0.5848 & 3 \\
\hline & {$[0.35,0.45]$} & 0.4084 & 4 & & {$[0.45,0.55]$} & 0.5000 & 1 & & {$[0.55,0.65]$} & 0.5916 & 2 \\
\hline & {$[0.40,0.40]$} & 0.4000 & 5 & & {$[0.50,0.50]$} & 0.5000 & 1 & & {$[0.60,0.60]$} & 0.6000 & 1 \\
\hline \multirow{5}{*}{$\epsilon=0.05 * r_{3}$} & {$[0.20,0.60]$} & 0.4247 & 1 & & {$[0.30,0.70]$} & 0.5000 & 1 & \multirow{5}{*}{$\epsilon=0.05 * r_{3}$} & {$[0.40,0.80]$} & 0.5753 & 5 \\
\hline & {$[0.25,0.55]$} & 0.4206 & 2 & & {$[0.35,0.65]$} & 0.5000 & 1 & & {$[0.45,0.75]$} & 0.5794 & 4 \\
\hline & {$[0.30-\epsilon, 0.50+\epsilon]$} & 0.4164 & 3 & $\epsilon=0.05 * r_{3}$ & {$[0.40-\epsilon, 0.60+\epsilon]$} & 0.5000 & 1 & & {$[0.50-\epsilon, 0.70+\epsilon]$} & 0.5836 & 3 \\
\hline & {$[0.35,0.45]$} & 0.4084 & 4 & & {$[0.45,0.55]$} & 0.5000 & 1 & & {$[0.55,0.65]$} & 0.5916 & 2 \\
\hline & {$[0.40,0.40]$} & 0.4000 & 5 & & {$[0.50,0.50]$} & 0.5000 & 1 & & {$[0.60,0.60]$} & 0.6000 & 1 \\
\hline \multirow{5}{*}{$\epsilon=0.05 * r_{4}$} & {$[0.20,0.60]$} & 0.4246 & 1 & & {$[0.30,0.70]$} & 0.5000 & 1 & \multirow{5}{*}{$\epsilon=0.05 * r_{4}$} & {$[0.40,0.80]$} & 0.5754 & 5 \\
\hline & {$[0.25,0.55]$} & 0.4206 & 2 & & {$[0.35,0.65]$} & 0.5000 & 1 & & {$[0.45,0.75]$} & 0.5794 & 4 \\
\hline & {$[0.30,0.50]$} & 0.4151 & 3 & $\epsilon=0.05 * r_{4}$ & {$[0.40,0.60]$} & 0.5000 & 1 & & {$[0.50,0.70]$} & 0.5849 & 3 \\
\hline & {$[0.35-\epsilon, 0.45+\epsilon]$} & 0.4091 & 4 & & {$[0.45-\epsilon, 0.55+\epsilon]$} & 0.5000 & 1 & & {$[0.55-\epsilon, 0.65+\epsilon]$} & 0.5909 & 2 \\
\hline & {$[0.40,0.40]$} & 0.4000 & 5 & & {$[0.50,0.50]$} & 0.5000 & 1 & & {$[0.60,0.60]$} & 0.6000 & 1 \\
\hline \multirow{5}{*}{$\epsilon=0.05 * r_{5}$} & {$[0.20,0.60]$} & 0.4245 & 1 & & {$[0.30,0.70]$} & 0.5000 & 1 & \multirow{5}{*}{$\epsilon=0.05 * r_{5}$} & {$[0.40,0.80]$} & 0.5755 & 5 \\
\hline & {$[0.25,0.55]$} & 0.4205 & 2 & & {$[0.35,0.65]$} & 0.5000 & 1 & & {$[0.45,0.75]$} & 0.5795 & 4 \\
\hline & {$[0.30,0.50]$} & 0.4151 & 3 & $\epsilon=0.05 * r_{5}$ & {$[0.40,0.60]$} & 0.5000 & 1 & & {$[0.50,0.70]$} & 0.5849 & 3 \\
\hline & {$[0.35,0.45]$} & 0.4083 & 4 & & {$[0.45,0.55]$} & 0.5000 & 1 & & {$[0.55,0.65]$} & 0.5917 & 2 \\
\hline & {$[0.40-\epsilon, 0.40+\epsilon]$} & 0.4000 & 5 & & {$[0.50-\epsilon, 0.50+\epsilon]$} & 0.5000 & 1 & & {$[0.60-\epsilon, 0.60+\epsilon]$} & 0.6000 & 1 \\
\hline
\end{tabular}

Furthermore, our proposed method would also be suitable for the real-life scenarios, by comparing with existing methods in the interval-valued environment. For instance, Gitinavard et al. [67] introduced a novel interval-valued hesitant fuzzy group outranking approach, which has been proved the feature of practicality for proceeding with the green supplier selection. Here, we utilized the example shown in [67] to carry out the comparative analysis process. In specific, three green suppliers $\left(A_{1}, A_{2}\right.$, and $\left.A_{3}\right)$ are required to be considered with five criteria which are shown as follows: 1) $C_{1}$ : cost; 2) $C_{2}$ : quality; 3) $C_{3}$ : delivery; 4) $C_{4}$ : technology capability; and 5) $C_{5}$ : environmental competency.

The normalized weights $\omega$ for all the criteria and the aggregated normalized interval-valued hesitant fuzzy decision matrix are shown in Tables X and XI, respectively.

Completed weighted averaging aggregation process, the ranking outcomes for all the green suppliers based on the proposed method are shown in Table XII.

Therefore, the second and third candidate green suppliers are considered as the best and worst selection, respectively, which is consistent with the results achieved in [67].

In Section V, the result of the MCDM problem with intervalvalued criteria satisfactions is discussed by comparing the parts, in which the order is needed in the decision process. In addition, the rationality of the proposed method can also be observed.

\section{Sensitivity Analysis}

In this section, we include a sensitivity analysis to indicate the robustness and sensitiveness of our proposed approach. At the initial step, we determine the parameter $\epsilon$ to express the possible error caused by experts. Then, with the data-driven principle, we assume that there are five intervals in comparison system where four of them are fixed. The parameter $\epsilon$ is constrained to be less than 0.05 , which can be regarded as a proper deviation for error factor.

The five intervals in case 1 are determined as $V_{1}=$ $[0.20,0.60], V_{2}=[0.25,0.55], V_{3}=[0.30,0.50], V_{4}=$ $[0.35,0.45]$, and $V_{5}=[0.40,0.40]$. We note that the midpoints of all of them are 0.4 . In terms of case 2, all of the intervals' midpoints are fixed as 0.5 . Then, we note all the midpoints of intervals shown in case 3 are 0.6. In this case, if we simply use the midpoint method to measure its size, the effective ranking outcomes cannot be achieved. Due to the different error level reflected by different $\epsilon$ values, the calculation results of its GGRVs are shown as follows.

As shown in Table XIII, we note the ranking outcomes cannot be changed with the introduction of the error factor, suggesting the robustness, and sensitiveness of our proposed approach. Specifically, considering that the range of unit interval is 1 , the range values of intervals within the unit interval are usually less than 1 . Thus, the difference between the left and right endpoints of our selected five intervals is constantly set to 0.05 , which indicates that the error factor cannot take an influence on the uncertainty relation of such intervals in comparison system. Furthermore, the ranking outcomes reflecting the features of the golden rule are shown in Section III.

\section{CONCLUSION}

In this paper, we proposed a novel approach to order the intervals based on the golden rule. This approach introduces 
the modified formulas, definitions, and parameters, which considers the uncertainty information created by the range value and intervals' effects in an information system. The goal of this approach is to improve the MCDM problems when the satisfaction for all the alternatives are interval valued. As the traditional midpoint method cannot develop a model to deal with the uncertainty information, our proposed approach is a complementary model to improve the uncertainties related problems. Our results show the greater the uncertainty and the smaller their representative values for the intervals in a comparison system. This finding is consistent with the realistic MCDM scenario.

It should be noted that there are still some limitations of the proposed method. For instance, to illustrate the problem, the proposed method has not been applied in solving more complex decision situations. Moreover, we believe that it can be used to solve other kinds of problems. This issue is one of our ongoing works.

\section{ACKNOWLEDGMENT}

The authors would like to thank the reviewers' suggestions and the editor's encouragement.

\section{REFERENCES}

[1] H. Zhang, Y. Dong, and X. Chen, "The 2-rank consensus reaching model in the multigranular linguistic multiple-attribute group decision-making," IEEE Trans. Syst., Man, Cybern., Syst., vol. 48, no. 12, pp. 2080-2094, Dec. 2018.

[2] C. Fu, D.-L. Xu, and S.-L. Yang, "Distributed preference relations for multiple attribute decision analysis," J. Oper. Res. Soc., vol. 67, no. 3, pp. 457-473, 2016.

[3] R. R. Yager, "Categorization in multi-criteria decision making," Inf. Sci., vols. 460-461, pp. 416-423, Sep. 2018.

[4] L. Fei, Y. Deng, and Y. Hu, "DS-VIKOR: A new multi-criteria decisionmaking method for supplier selection," Int. J. Fuzzy Syst., vol. 21, no. 1, pp. $157-175,2019$.

[5] M. P. Borujeni and H. Gitinavard, "Evaluating the sustainable mining contractor selection problems: An imprecise last aggregation preference selection index method," J. Sustain. Min., vol. 16, no. 4, pp. 207-218, 2017.

[6] H. Zhang and Y. Deng, "Engine fault diagnosis based on sensor data fusion considering information quality and evidence theory," Adv. Mech. Eng., vol. 10, no. 11, pp. 1-10, 2018. doi: 10.1177/1687814018809184.

[7] Y. Li and Y. Deng, "Generalized ordered propositions fusion based on belief entropy," Int. J. Comput. Commun. Control, vol. 13, no. 5, pp. 792-807, 2018.

[8] F. Xiao and W. Ding, "Divergence measure of Pythagorean fuzzy sets and its application in medical diagnosis," Appl. Soft Comput., vol. 79, pp. 254-267, Jun. 2019.

[9] C.-T. Lin et al., "Forehead EEG in support of future feasible personal healthcare solutions: Sleep management, headache prevention, and depression treatment," IEEE Access, vol. 5, pp. 10612-10621, 2017.

[10] E. Huang, S. Zhang, L. H. Lee, E. P. Chew, and C.-H. Chen, "Improving analytic hierarchy process expert allocation using optimal computing budget allocation," IEEE Trans. Syst., Man, Cybern., Syst., vol. 46, no. 8 , pp. 1140-1147, Aug. 2016.

[11] X. Deng and Y. Deng, "D-AHP method with different credibility of information," Soft Comput., vol. 23, no. 2, pp. 683-691, 2019.

[12] S. Ebrahimnejad, H. Gitinavard, and S. Sohrabvandi, "A new extended analytical hierarchy process technique with incomplete interval-valued information for risk assessment in IT outsourcing," Int. J. Eng., vol. 30, no. 5, pp. 739-748, May 2017.

[13] H. Xu and Y. Deng, "Dependent evidence combination based on decision-making trial and evaluation laboratory method," Int. J. Intell. Syst., vol. 34, no. 7, pp. 1555-1571, 2019. doi: 10.1002/int.22107.

[14] X. Zhou, Y. Hu, Y. Deng, F. T. S. Chan, and A. Ishizaka, "A DEMATELbased completion method for incomplete pairwise comparison matrix in AHP," Ann. Oper. Res., vol. 271, no. 2, pp. 1045-1066, 2018.
[15] H. Gitinavard, M. S. Pishvaee, and F. Jalalvand, "A hierarchical multicriteria group decision-making method based on TOPSIS and hesitant fuzzy information," Int. J. Appl. Decis. Sci., vol. 10, no. 3, pp. 213-232, 2017.

[16] L. Zhang, Y. Xu, C.-H. Yeh, L. He, and D.-Q. Zhou, "Bi-TOPSIS: A new multicriteria decision making method for interrelated criteria with bipolar measurement," IEEE Trans. Syst., Man, Cybern., Syst., vol. 47, no. 12 , pp. 3272-3283, Dec. 2017.

[17] H. Gitinavard, S. M. Mousavi, and B. Vahdani, "Soft computingbased new interval-valued hesitant fuzzy multi-criteria group assessment method with last aggregation to industrial decision problems," Soft Comput., vol. 21, no. 12, pp. 3247-3265, Jun. 2017.

[18] D. Han, J. Dezert, and Y. Yang, "Belief interval-based distance measures in the theory of belief functions," IEEE Trans. Syst., Man, Cybern., Syst., vol. 48, no. 6, pp. 833-850, Jun. 2018.

[19] C. Fu, D.-L. Xu, and M. Xue, "Determining attribute weights for multiple attribute decision analysis with discriminating power in belief distributions," Knowl. Based Syst., vol. 143, no. 1, pp. 127-141, Mar. 2018.

[20] L. Yin, X. Deng, and Y. Deng, "The negation of a basic probability assignment," IEEE Trans. Fuzzy Syst., vol. 27, no. 1, pp. 135-143, Jan. 2019.

[21] F. Xiao, "A multiple-criteria decision-making method based on $D$ numbers and belief entropy," Int. J. Fuzzy Syst., vol. 21, no. 4, pp. 1144-1153, 2019 .

[22] F. Xiao, "Multi-sensor data fusion based on the belief divergence measure of evidences and the belief entropy," Inf. Fusion, vol. 46, no. 2019, pp. 23-32, Mar. 2019.

[23] H. Gitinavard and M. A. Shirazi, "An extended intuitionistic fuzzy modified group complex proportional assessment approach," J. Ind. Syst. Eng., vol. 11, no. 3, pp. 229-246, 2018.

[24] P. Gupta, C.-T. Lin, M. K. Mehlawat, and N. Grover, "A new method for intuitionistic fuzzy multiattribute decision making," IEEE Trans. Syst., Man, Cybern., Syst., vol. 46, no. 9, pp. 1167-1179, Sep. 2016.

[25] G.-W. Wei, "GRA method for multiple attribute decision making with incomplete weight information in intuitionistic fuzzy setting," Knowl. Based Syst., vol. 23, no. 3, pp. 243-247, 2010.

[26] H. Gitinavard and M. H. F. Zarandi, "A mixed expert evaluation system and dynamic interval-valued hesitant fuzzy selection approach," Int. J. Math. Comput. Phys. Elect. Comput. Eng., vol. 10, pp. 337-345, 2016.

[27] R. R. Yager and N. Alajlan, "Multi-criteria formulations with uncertain satisfactions," Eng. Appl. Artif. Intell., vol. 69, pp. 104-111, Mar. 2018.

[28] A. Shahpari and S. A. Seyedin, "Using mutual aggregate uncertainty measures in a threat assessment problem constructed by DempsterShafer network," IEEE Trans. Syst., Man, Cybern., Syst., vol. 45, no. 6, pp. 877-886, Jun. 2015.

[29] F.-Y. Meng, Q.-X. An, C.-Q. Tan, and X.-H. Chen, "An approach for group decision making with interval fuzzy preference relations based on additive consistency and consensus analysis," IEEE Trans. Syst., Man, Cybern., Syst., vol. 47, no. 8, pp. 2069-2082, Aug. 2017.

[30] A. Halder et al., "General and interval type-2 fuzzy face-space approach to emotion recognition," IEEE Trans. Syst., Man, Cybern., Syst., vol. 43, no. 3, pp. 587-605, May 2013.

[31] Y. Han, Y. Deng, Z. Cao, and C.-T. Lin, "An interval-valued pythagorean prioritized operator-based game theoretical framework with its applications in multicriteria group decision making," Neural Comput. Appl., pp. 1-19, 2019. doi: 10.1007/s00521-019-04014-1.

[32] M. Öztürk and A. Tsoukiàs, "A valued Ferrers relation for interval comparison," Fuzzy Sets Syst., vol. 266, pp. 47-66, May 2015.

[33] A. Bufardi, "An alternative definition for fuzzy interval orders," Fuzzy Sets Syst., vol. 133, no. 2, pp. 249-259, 2003.

[34] S. Ezadi, T. Allahviranloo, and S. Mohammadi, "Two new methods for ranking of $z$-numbers based on sigmoid function and sign method," Int. J. Intell. Syst., vol. 33, no. 7, pp. 1476-1487, 2018.

[35] H. Bustince, J. Fernandez, A. Kolesárová, and R. Mesiar, "Generation of linear orders for intervals by means of aggregation functions," Fuzzy Sets Syst., vol. 220, pp. 69-77, Jun. 2013.

[36] R. R. Yager, "Golden rule representative values for non-standard membership grades," in Proc. IEEE 8th Int. Conf. Intell. Syst., vol. 26. Sofia, Bulgaria, 2016, pp. 2-7.

[37] R. R. Yager, "Golden rule and other representative values for Atanassov type intuitionistic membership grades," IEEE Trans. Fuzzy Syst., vol. 23, no. 6, pp. 2260-2269, Dec. 2015.

[38] C. Kahraman, Fuzzy Multi-Criteria Decision Making: Theory and Applications With Recent Developments (Springer Series in Optimization and Its Applications), vol. 16. New York, NY, USA: Springer, 2008, pp. $1-591$. 
[39] R. R. Yager, "Multi-criteria decision making with interval criteria satisfactions using the golden rule representative value," IEEE Trans. Fuzzy Syst., vol. 26, no. 2, pp. 1023-1031, Apr. 2018.

[40] J. Han and C. Moraga, "The influence of the sigmoid function parameters on the speed of backpropagation learning," in From Natural to Artificial Neural Computation, J. Mira and F. Sandoval, Eds. Heidelberg, Germany: Springer, 1995, pp. 195-201.

[41] T. Takagi and M. Sugeno, "Fuzzy identification of systems and its applications to modeling and control," IEEE Trans. Syst., Man, Cybern., vol. 15, no. 1, pp. 116-132, Jan./Feb. 1985.

[42] X. Gu, F.-L. Chung, H. Ishibuchi, and S. Wang, "Imbalanced TSK fuzzy classifier by cross-class Bayesian fuzzy clustering and imbalance learning," IEEE Trans. Syst., Man, Cybern., Syst., vol. 47, no. 8, pp. 2005-2020, Aug. 2017.

[43] Y. Jiang, Z. Deng, F.-L. Chung, and S. Wang, "Realizing two-view TSK fuzzy classification system by using collaborative learning," IEEE Trans. Syst., Man, Cybern., Syst., vol. 47, no. 1, pp. 145-160, Jan. 2017.

[44] T. Zhou, H. Ishibuchi, and S. Wang, "Stacked blockwise combination of interpretable TSK fuzzy classifiers by negative correlation learning," IEEE Trans. Fuzzy Syst., vol. 26, no. 6, pp. 3327-3341, Dec. 2018.

[45] X. Gu and S. Wang, "Bayesian Takagi-Sugeno-Kang fuzzy model and its joint learning of structure identification and parameter estimation," IEEE Trans. Ind. Informat., vol. 14, no. 12, pp. 5327-5337, Dec. 2018.

[46] J. R. Castro, O. Castillo, P. Melin, and A. Rodríguez-Díaz, "A hybrid learning algorithm for a class of interval type-2 fuzzy neural networks," Inf. Sci., vol. 179, no. 13, pp. 2175-2193, 2009.

[47] K. Lapa, K. Cpalka, and L. Rutkowski, "New aspects of interpretability of fuzzy systems for nonlinear modeling," in Advances in Data Analysis With Computational Intelligence Methods. Cham, Switzerland: Springer, 2018, pp. 225-264.

[48] M. Li, Q. Zhang, and Y. Deng, "Evidential identification of influential nodes in network of networks," Chaos Solitons Fractals, vol. 117, pp. 283-296, Dec. 2018.

[49] Q. Li, J. Zhong, Q. Li, C. Wang, and Z. Cao, "A community merger of optimization algorithm to extract overlapping communities in networks," IEEE Access, vol. 7, pp. 3994-4005, 2019.

[50] H. Yang, Y. Deng, and J. Jones, "Network division method based on cellular growth and physarum-inspired network adaptation," Int. J. Unconv. Comput., vol. 13, no. 6, pp. 477-491, 2018.

[51] A. Slowik and H. Kwasnicka, "Introduction to the special section on nature inspired methods in industry applications," IEEE Trans. Ind. Informat., vol. 14, no. 3, pp. 1001-1003, Mar. 2018.

[52] K. Łapa and K. Cpałka, "Flexible fuzzy PID controller (FFPIDC) and a nature-inspired method for its construction," IEEE Trans. Ind. Informat. vol. 14, no. 3, pp. 1078-1088, Mar. 2018.

[53] A. Slowik and H. Kwasnicka, "Nature inspired methods and their industry applications-Swarm intelligence algorithms," IEEE Trans. Ind. Informat., vol. 14, no. 3, pp. 1004-1015, Mar. 2018.

[54] J. Chrol-Cannon, Y. Jin, and A. Grüning, "An efficient method for online detection of polychronous patterns in spiking neural networks," Neurocomputing, vol. 267, pp. 644-650, Dec. 2017.

[55] Z.-H. Cao, L.-W. Ko, K.-L. Lai, S.-B. Huang, S.-J. Wang, and C.-T. Lin, "Classification of migraine stages based on resting-state EEG power," in Proc. Int. Joint Conf. Neural Netw. (IJCNN), Killarney, Ireland, Jul. 2015, pp. 1-5.

[56] T. J. Ross, Fuzzy Logic With Engineering Applications, vol. 2. Hoboken, NJ, USA: Wiley, 1995.

[57] J. Botzheim and P. Földesi, "Novel calculation of fuzzy exponent in the sigmoid functions for fuzzy neural networks," Neurocomputing, vol. 129 , pp. 458-466, Apr. 2014.

[58] M. R. Zadeh, S. Amin, D. Khalili, and V. P. Singh, "Daily outflow prediction by multi layer perceptron with logistic sigmoid and tangent sigmoid activation functions," Water Resour. Manag., vol. 24, no. 11, pp. 2673-2688, Sep. 2010.

[59] L. Hui, "Sigmoid-linear BP neural network based variable selecting method," in Proc. 5th Int. Conf. Nat. Comput. (ICNC), vol. 2. Tianjin, China, Aug. 2009, pp. 434-438.

[60] K.-A. Toh, "Training a reciprocal-sigmoid classifier by feature scalingspace," Mach. Learn., vol. 65, no. 1, pp. 273-308, 2006.

[61] G.-Z. Cao, N. Chen, S.-D. Huang, S.-S. Xiao, and J. He, "Nonlinear modeling of the flux linkage in 2-D plane for the planar switched reluctance motor," IEEE Trans. Magn., vol. 54, no. 11, pp. 1-5, Nov. 2018.

[62] S. Raghu and N. Sriraam, "Optimal configuration of multilayer perceptron neural network classifier for recognition of intracranial epileptic seizures," Expert Syst. Appl., vol. 89, pp. 205-221, Dec. 2017.
[63] Z. Chen and F. Cao, "The approximation operators with sigmoidal functions," Comput. Math. Appl., vol. 58, no. 4, pp. 758-765, 2009.

[64] Z. Tao, X. Liu, H. Chen, and L. Zhou, "Using new version of extended t-norms and s-norms for aggregating interval linguistic labels," IEEE Trans. Syst., Man, Cybern., Syst., vol. 47, no. 12, pp. 3284-3298, Dec. 2017.

[65] E. Pap, "Theory and applications of non-additive measures and corresponding integrals," in Proc. 10th Int. Conf. Model. Decis. Artif. Intell. (MDAI), Barcelona, Spain, Nov. 2013, pp. 1-10.

[66] R. R. Yager, "Multicriteria decision-making using fuzzy measures," Cybern. Syst., vol. 46, nos. 3-4, pp. 150-171, 2015.

[67] H. Gitinavard, H. Ghaderi, and M. S. Pishvaee, "Green supplier evaluation in manufacturing systems: A novel interval-valued hesitant fuzzy group outranking approach," Soft Comput., vol. 22, no. 19, pp. 6441-6460, Oct. 2018 .

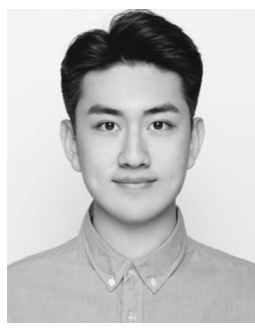

Zeyi Liu is currently pursuing the bachelor's degree in computer science and technology with the School of Computer and Information Science, Southwest University, Chongqing, China.

His current research interests include multicriteria decision analysis, information fusion, and fuzzy logic.

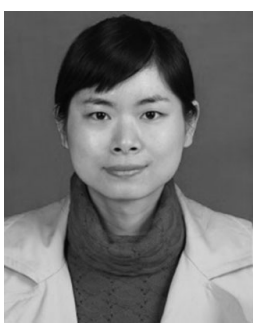

Fuyuan Xiao received the D.E. degree in computer science and communication engineering from the Graduate School of Science and Technology, Kumamoto University, Kumamoto, Japan, in 2014.

Since 2014, she has been with the School of Computer and Information Science, Southwest University, Chongqing, China, where she is an Associate Professor. She has published over 30 papers in the prestigious journals and conferences, including Information Fusion, Applied Soft Computing, Engineering Applications of Artificial Intelligence, IEICE Transactions on Information and Systems, and Artificial Intelligence in Medicine. Her current research interests include information fusion, distributed data stream processing, complex event processing, and quantum information processing.

Dr. Xiao severs as a Reviewer in the prestigious journals, such as the IEEE Transactions on FuZzy Systems, Information Sciences, KnowledgeBased Systems, Engineering Applications of Artificial Intelligence, Future Generation Computer Systems, Reliability Engineering and System Safety, and Artificial Intelligence in Medicine.

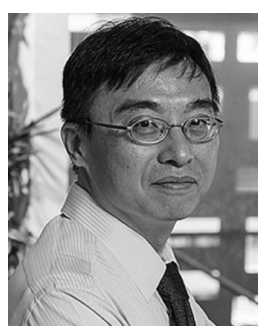

Chin-Teng Lin (F'03) received the B.S. degree in electrical control engineering from National Chiao Tung University, Hsinchu, Taiwan, in 1986, and the master's and Ph.D. degrees in electrical engineering from Purdue University, West Lafayette, IN, USA, in 1989 and 1992, respectively.

$\mathrm{He}$ is currently a Distinguished Professor with the University of Technology Sydney, Sydney, NSW, Australia, and holds an Honorary Professorship with the University of Nottingham, Nottingham, U.K. He has coauthored a book entitled Neural Fuzzy Systems (Prentice Hall) and has written a book entitled Neural Fuzzy Control Systems With Structure and Parameter Learning (World Scientific). He has authored over 200 journal articles (Total Citation: $18438, H$-index: 51, and i10-index: 301), including 97 IEEE journal articles, in the areas of neural networks, fuzzy systems, multimedia hardware/software, and cognitive neuroengineering.

Dr. Lin has been serving as the Editor-in-Chief for the IEEE TRANSACTIONS ON FuZZY Systems since 2011. He served on the Board of Governors of the IEEE Circuits and Systems (CAS) Society from 2005 to 2008, the IEEE Systems, Man, Cybernetics Society from 2003 to 2005, and the IEEE Computational Intelligence Society from 2008 to 2010, and was the Chair of the IEEE Taipei Section from 2009 to 2010. He was a Distinguished Lecturer in the IEEE CAS Society from 2003 to 2005 . He served as the Deputy Editor-in-Chief of the IEEE TRANSACTIONS ON CIRCUITS AND SYSTEMSPART II: EXPRESS BRIEFS from 2006 to 2008. He was the Program Chair of the IEEE International Conference on Systems, Man, and Cybernetics in 2005 and the General Chair of the 2011 IEEE International Conference on Fuzzy Systems. He was a fellow of the International Fuzzy Systems Association in 2012. 


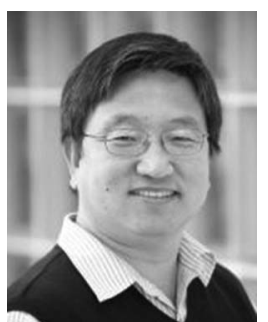

Byeong Ho Kang (SM'05) received the Ph.D. degree in computer science from the University of New South Wales, Sydney, NSW, Australia, in 1996.

$\mathrm{He}$ is a Professor with the School of Engineering and ICT, University of Tasmania, Hobart, TAS, Australia, where he leads the Smart Services and Systems Research Group of post-doctoral scientists which has carried out fundamental and applied research in research areas, expert systems, Web services, SNS analysis, and smart industry areas. He was a Visiting Researcher with the Advanced Research Laboratory, HITACHI, Hitachi, Japan. He has also taken part in major research and development projects with industries and research organizations, including the Smart Internet Collaborative Research Centre, the U.S. Air Force, and Hyundai Steel.

Dr. Kang has served as the chair and a steering committee member in many international organizations and during conferences.

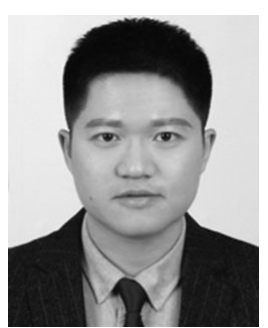

Zehong Cao (M'17) received the B.S. degree in electronic and information engineering from Northeastern University, Shenyang, China, in 2012, the M.S. degree in electronics engineering from the Chinese University of Hong Kong, Hong Kong, in 2013, the first Ph.D. degree in information technology from the University of Technology Sydney (UTS), Sydney, NSW, Australia, and the second $\mathrm{Ph} . \mathrm{D}$. degree in electrical and control engineering from National Chiao Tung University (NCTU), Hsinchu, Taiwan, in 2017.

$\mathrm{He}$ is a Lecturer (also known as Assistant Professor) with the Discipline of Information and Communication Technology, School of Technology, Environments and Design, College of Sciences and Engineering, University of Tasmania, Hobart, TAS, Australia, and an Adjunct Fellow with the School of Computer Science, Faculty of Engineering and Information Technology, UTS. He had an ESI highly cited paper in 2019 and a string of successful over 30 publications among the most respected journals, including Nature Scientific Data, IEEE Transactions on FuZZy Systems, IEEE TransaCtions ON NEURAL NETWORKS AND LEARNING SySTEMS, IEEE TRANSACTIONS ON CybERnETICS, IEEE TRANSACTIONS ON Systems, MAN, AND CyBERNeTiCS: SySTEMS, IEEE Systems, Man, and Cybernetics Magazine, IEEE Transactions on Biomedical Engineering, IEEE ACCESS, International Journal of Neural Systems, Information Science, Neurocomputing, and Neural Computing and Applications. His current research interests include fuzzy sets and systems, fuzzy neural networks, brain-computer interaction, biosignal processing, game-based machine learning, and data mining.

Dr. Cao was a recipient of the UTS Centre for Artificial Intelligence Best Paper Award in 2017, the UTS Faculty of Engineering and IT Publication Award in 2017, the UTS President Scholarship in 2015, and the NCTU \& Songshanhu Scholarship in 2013. He has been serving as an Associate Editor for IEEE ACCESS since 2018 and Journal of Intelligent and Fuzzy Systems since 2019. He was a Guest Editor of the IEEE TRANSACTIONS ON EMERging Topics in Computational InTElligence in 2019, Swarm and Evolutionary Computation in 2019, and Neurocomputing in 2018. 